\title{
Effects of Different Mineral Supplements on Fertilization of Phenol-contaminated Soils by Corynebacterium glutamicum
}

\author{
Bit-Na Kim, Thai-Hoang Le, EunSeon Hong, Joo-Myung Ahn, Yang-Hoon Kim, and Jiho Min
}

Received: 26 November 2013 / Revised: 24 February 2014 / Accepted: 1 March 2014

(C) The Korean Society for Biotechnology and Bioengineering and Springer 2014

In the 2014 issue of Biotechnology and Bioprocess Engineering (BBE), an error occurred in the research article: Bit-Na Kim, Thai-Hoang Le, EunSeon Hong, Joo-Myung Ahn, YangHoon Kim, and Jiho Min (2014) Effects of Different Mineral Supplements on Fertilization of Phenol-contaminated Soils by Corynebacterium glutamicum. Biotechnol. Bioprocess Eng. 19: 276-281.

\section{- In the authors's affiliation}

\section{Original:}

Yang-Hoon Kim

Department of Civil and Environmental Engineering, Hannam University, Daejon 306-791, Korea

This should be replaced by:

Yang-Hoon Kim

Department of Microbiology, Chungbuk National University, Cheongju 361-763, Korea

Received: 26 May 2014
Bit-Na Kim, EunSeon Hong, Joo-Myung Ahn, Jiho Min

Graduate School of Semiconductor and Chemical Engineering, Chonbuk National University, Jeonju 561-756, Korea

Thai-Hoang Le, Jiho Min*

Department of Bioprocess Engineering, Chonbuk National University, Jeonju 561-756, Korea

Tel: +82-63-270-2436; Fax: +82-63-270-2306

E-mail: jihomin@jbnu.ac.kr

Yang-Hoon Kim

Department of Microbiology, Chungbuk National University, Cheongju 361-763, Korea 